\title{
The Characterization of Differential Operators by Locality: Dissipations and Ellipticity
}

\author{
By
}

Ola Bratteli*, George A. ElliotT** and Derek W. Robinson***

\begin{abstract}
Let $\delta$ be the generator of a $C_{0}$-group of *automorphisms of a $C^{*}$-algebra $A$ and $H$ a differential operator of the form

$$
H=\sum_{m=0}^{p} \lambda_{m} \grave{0}^{m},
$$

where $\lambda_{m} \in C$. It is known from a previous work that if $\mathcal{A}$ is abelian then $H$ is a dissipation, i.e.

$$
H\left(a^{*} a\right) \leqq a^{*} H(a)+H\left(a^{*}\right) a, \quad a \in D(H),
$$

if, and only if, $\lambda_{m}=0$ for $m>2, \lambda_{2} \leqq 0$, and $\lambda_{0} \geqq 0$. This conclusion is no longer generally true for non-abelian $\mathcal{A}$, but it is true in a variety of special cases which we discuss, e.g. if $\mathcal{A}$ is isomorphic to the $C^{*}$-algebra of all compact operators on a Hilbert space $\mathcal{A}$ and $\delta$ is unbounded.
\end{abstract}

\section{$\S 0$. Introduction}

Let $\delta$ denote a symmetric derivation on a $C *$-algebra $A$ and consider the differential operators $H: A_{\infty} \rightarrow \mathcal{A}$ where

$$
\mathcal{A}_{\infty}=\bigcap_{n \geq 1} D\left(\delta^{n}\right)
$$

and

$$
H=\sum_{m=0}^{p} \lambda_{m} \delta^{m}, \quad \lambda_{m} \in C .
$$

If $p=2, \lambda_{0} \geqq 0$, and $\lambda_{2} \leqq 0$, then $H$ is a dissipation, i.e.

$$
H(a * a) \leqq H(a *) a+a * H(a), \quad a \in \mathcal{A}_{\infty} .
$$

The purpose of this note is to investigate conditions under which the converse is true.

Communicated by H. Araki, April 6, 1985.

* Research Institute for Mathematical Sciences, Kyoto University, Kyoto 606, Japan. Institute of Mathematics, University of Trondheim, N-7034, Trondheim-NTH, Norway.

** Mathematics Institute, University of Copenhagen, DK 2100 Copenhagen ø, Denmark.

*** Department of Mathematics, Institute of Advanced Studies, Australian National University, GPO Box 4, Canberra, ACT 2601, Australia. 
If $\mathcal{A}$ is finite-dimensional then there are certainly derivations $\delta$ and differential operators $H$ of this type which are dissipations but which are not quadratic in $\delta$. The simplest examples occur if $\mathcal{A}=M_{3}$, the $3 \times 3$ matrices.

Example 0.1. Define the derivation $\delta$ on $M_{3}$ by $\delta(a)_{\jmath l}=i k_{\jmath l} a_{\jmath l}$ where $k_{\jmath l}=$ $-k_{l j}$ and $k_{01}=1, k_{02}=1+\varepsilon, k_{12}=\varepsilon$. Next define $H$ by $H(a)_{\jmath l}=-h_{\jmath l}^{2} a_{\jmath l}$ where $h_{\jmath \iota}$ $=-h_{l j}$ and $h_{01}=1+2 \varepsilon, h_{02}=1-\varepsilon^{2}$, and $h_{12}=-\varepsilon(\varepsilon+2)$. Then $H$ is a dissipation. because it is the square of a derivation. Furthermore $H=\lambda_{2} \delta^{2}+\lambda_{4} \delta^{4}$ with $\lambda_{2}=$ $4\left(1+\varepsilon+\varepsilon^{2}\right)$ and $\lambda_{4}=3$. But unless $\varepsilon=0$ or $\varepsilon=-1$ one cannot express $H$ as a quadratic form in $\delta$. (If $\varepsilon=0$ or $\varepsilon=-1$ one has $H=-\delta^{2}$.)

Nevertheless if $\mathcal{A}$ is abelian it was established in [2] that the only differential operators $H$ which are dissipations are perforce quadratic. This is even the case if the coefficients $\lambda_{m}$ are not constant, e.g. if $\lambda_{m} \in \mathcal{A}$. A crucial feature in the abelian case is that if the derivation $\delta$ is non-zero then it is automatically unbounded, and it is quite possible that unboundedness of $\delta$ together with simplicity of $A$ is sufficient to ensure that only quadratic $H$ can be dissipations. Although we have not been able to prove this we will verify a variety of particular cases in Section 3. These all follow from estimations given in Section 2 which are based upon several spectral assumptions for $\delta$, which are mostly true if $\delta$ is unbounded and generates a one-parameter group of $*$-automorphisms of $\mathcal{A}$. Thus as motivation we begin, in Section 1, by examining the appropriate spectral properties of generators.

\section{$\S 1$. Spectral Properties}

In this section we assume that $\delta$ generates a strongly continuous one-parameter group $\tau$ of *automorphisms of $\mathcal{A}$ and examine spectral properties. For this we adopt the notation of [5], Section 3.2. The main result of this section, Proposition 1.1, is related to Connes' characterization, [6], Lemma 2.3.5, of the spectrum of $\tau$. Connes' argument can be slightly simplified by using the following known lemma. (This simplification is implicit in the proof of $2 \Rightarrow 3$ in [5], Proposition 3.2.40, but the argument there has a gap which is filled by the following lemma.)

Lemma 1.0. Let $\boldsymbol{G}$ be a locally compact abelian group with dual group $\hat{G}$, let $\gamma_{0} \in \hat{\boldsymbol{G}}$ and let $W$ be a neighborhood of $\gamma_{0}$ in $\hat{\boldsymbol{G}}$. Let $\left\{F_{s} ; s \in S\right\}$ be a collection of functions in $L^{1}(G)$ with the properties

1a. $\hat{F}_{s}\left(\gamma_{0}\right)=0$ for all $s \in S$, where ^ denotes Fourier transform.

1b. $\sup _{s \in S}\left\|F_{s}\right\|_{1}<+\infty$.

1c. For any $\delta>0$ there is a compact subset $E \subseteq G$ such that

$$
\int_{G \backslash E} d g\left|F_{s}(g)\right|<\delta
$$


for all $s \in S$.

It follows that for any $\varepsilon>0$ there exists $a g \in L^{1}(\boldsymbol{G})$ such that

2a. $\|g\|_{1}<1+\varepsilon$.

2b. $\hat{g}=1$ in a neighbourhood of $\gamma_{0}$ and $\hat{g}=0$ outside $W$.

2c. $\left\|F_{s} * g\right\|<\varepsilon$ for all $s \in S$.

Proof. In the case that $S$ is a one point set, this is Theorem 2.6.3 in [9]. The proof of the present generalization is almost identical with the original proof, and is therefore omitted.

In the following proposition, and later in the paper, we use the convention

$$
\hat{f}(k)=(2 \pi)^{-(1 / 2)} \int d t e^{2 k t} \hat{j}(t)
$$

for the Fourier transform on $\boldsymbol{R}$.

Proposition 1.1. For each $k \in \boldsymbol{R}, \boldsymbol{\varepsilon}>0$ and $p \in \mathbb{Z}$, there exists an $l>0$ such that

$$
\left\|\left.\left(\delta^{m}-(i k)^{m}\right)\right|_{\mathcal{A} \tau([k-l, k+l])}\right\|<\varepsilon, \quad m=1,2, \cdots, p .
$$

Proof. First choose an $f \in L^{1}(\boldsymbol{R})$ such that the Fourier transform is infinitely often differentiable with compact support and satisfies $\hat{f}=1$ on an interval $\left[k-l_{1}, k+l_{1}\right]$. This ensures, in particular, that $f$ is differentiable and $f^{\prime} \in L^{1}(\boldsymbol{R})$. Second, for each $s \in \mathbb{R}$ define $F_{s} \in L^{1}(\boldsymbol{R})$ by

One has

$$
F_{s}(t)= \begin{cases}\left(f(t-s)-e^{2 k s} f(t)\right) / s & \text { if } s \neq 0 \\ -f^{\prime}(t)-i k f(t) & \text { if } s=0 .\end{cases}
$$

$$
F_{s}(t)=(f(t-s)-f(t)) / s+f(t)\left(1-e^{2 k s}\right) / s
$$

for $s \neq 0$ and hence

$$
\left\|F_{s}\right\|_{1} \leqq\left\|f^{\prime}\right\|_{1}+k\|f\|_{1}
$$

for all $s \in \mathbb{R}$. Furthermore, $\hat{F}_{s}(k)=0$ for all $s$, and since $f \in \mathcal{S}(\mathbb{R})$ it is easy to see that condition $1 c$ is fulfilled if $s$ varies in the compact interval $S=[0,1]$. By Lemma 1.0 there is for each $\varepsilon^{\prime}>0$ a $g \in L^{1}(G)$ such that $\hat{g}=1$ on an interval $\left[k-l_{2}, k+l_{2}\right]$ and $\left\|F_{s} * g\right\|_{1}<\varepsilon^{\prime}$ for $s \in[0,1]$.

Next let $0<l<l_{2}$ and choose an $h \in L^{1}(\boldsymbol{R})$ with $\operatorname{supp}(\hat{h}) \subseteq\left[k-l_{2}, k+l_{2}\right]$ such that $\hat{h}=1$ on $[k-l, k+l]$. Then $\tau(h)(a)=a$ for all $a \in \mathcal{A}^{\tau}([k-l, k+l])$. Moreover for $s \in[0,1]$ and $p \in[k-l, k+l]$ one has

$$
\begin{aligned}
\left(F_{s} * g * h\right)^{\wedge}(p) & =\left(e^{\imath p s}-e^{\imath k s}\right) \hat{f}(p) \hat{g}(\not) \hat{h}(p), s \\
& =\left(e^{\imath p s}-e^{\imath k s}\right) \hat{h}(p) / s .
\end{aligned}
$$

Therefore if $a \in \mathcal{A}^{5}([k-l, k-l])$ one has 


$$
\begin{aligned}
\left\|\left(\tau_{s}(a)-e^{i k s} a\right) / s\right\| & =\left\|\left(\tau_{s}(\tau(h)(a))-e^{i k s} \tau(h)(a)\right) / s\right\| \\
& =\left\|\tau\left(F_{s} * g\right)(\tau(h)(a))\right\| \\
& \leqq\left\|F_{s} * g\right\|_{1}\|\tau(h)(a)\| \leqq \varepsilon^{\prime}\|a\| .
\end{aligned}
$$

Consequently in the limit $s \rightarrow 0$ one obtains

$$
\|\delta(a)-(i k) a\| \leqq \varepsilon^{\prime}\|a\| .
$$

Next note that if $a \in \mathcal{A}^{\tau}([k-l, k+l])$ then $\delta^{m}(a) \in \mathcal{A}^{\tau}([k-l, k+l])$ for all $m=$ $1,2, \cdots$. Hence

which implies

$$
\left\|\delta^{m+1}(a)-(i k) \delta^{m}(a)\right\| \leqq \varepsilon^{\prime}\left\|\delta^{m}(a)\right\|
$$

$$
\begin{aligned}
\left\|\delta^{m}(a)-(i k)^{m} a\right\| & \leqq \sum_{q=0}^{m-1}|k|^{q}\left\|\delta^{m-q}(a)-(i k) \delta^{m-q-1}(a)\right\| \\
& \leqq \varepsilon^{\prime} \sum_{q=0}^{m-1}|k|^{q}\left\|\delta^{m-q-1}(a)\right\|
\end{aligned}
$$

Moreover

$$
\begin{aligned}
\left\|\delta^{r}(a)\right\| & \leqq\left\|\delta^{r}(a)-(i k) \delta^{r-1}(a)\right\|+|k|\left\|\delta^{r-1}(a)\right\| \\
& \leqq\left(|k|+\varepsilon^{\prime}\right)\left\|\delta^{r-1}(a)\right\| \\
& \leqq\left(|k|+\varepsilon^{\prime}\right)^{r}\|a\| .
\end{aligned}
$$

Combining these estimates one calculates that

$$
\left\|\delta^{m}(a)-(i k)^{m} a\right\| \leqq\left(\left(|k|+\varepsilon^{\prime}\right)^{m}-|k|^{m}\right)\|a\| .
$$

Hence choosing $\varepsilon^{\prime}$ sufficiently small that

$$
\max \left\{\left(|k|+\varepsilon^{\prime}\right)^{m}-|k|^{m} ; m=1,2, \cdots, p\right\}<\varepsilon
$$

one obtains the Proposition.

Remark 1.2. If $a \in \mathcal{A}^{\tau}\left(\left[k-l_{1}, k+l_{1}\right]\right)$ and $b \in \mathcal{A}^{*}\left(\left[l-l_{1}, l+l_{1}\right]\right)$ then $a * a \in$ $\mathcal{A}^{\tau}\left(\left[-2 l_{1}, 2 l_{1}\right]\right), b * b \in \mathcal{A}^{\tau}\left(\left[-2 l_{1}, 2 l_{1}\right]\right)$, and $a^{*} b \in \mathcal{A}^{\pi}\left(\left[l-k-2 l_{1}, l-k+2 l_{1}\right]\right)$. Therefore given $\varepsilon>0$ and $p \in Z_{+}$one can choose $l_{1}$ sufficiently small that

$$
\begin{gathered}
\left\|\delta^{m}(a)-(i k)^{m} a\right\|<\varepsilon\|a\|,\left\|\delta^{m}(b)-(i l)^{m} b\right\|<\varepsilon\|b\|, \\
\left\|\delta^{m}(a * a)\right\|<\varepsilon\|a * a\|,\left\|\delta^{m}(b * b)\right\|<\varepsilon\|b * b\|, \\
\left\|\delta^{m}(a * b)-(i(l-k))^{m} a * b\right\|<\varepsilon\|a * b\|
\end{gathered}
$$

for $m=1,2, \cdots, p$.

Remark 1.3. Let $k$ be in the spectrum of $\delta$. Then for any $n$ there is an $a_{n} \in \mathcal{A}^{\tau}\left(\left[k-\frac{1}{n}, k+\frac{1}{n}\right]\right)$ with $\left\|a_{n}\right\|=1$. Put $c_{n}=a_{n} a_{n}^{*}$. Thus $c_{n} \in \mathcal{A}^{\tau}\left(\left[-\frac{2}{n}, \frac{2}{n}\right]\right)$ 
and $\left\|c_{n}\right\|=1, c_{n} \geqq 0$, and by Proposition 1.1

$$
\lim _{n \rightarrow \infty} \frac{\left\|\delta^{m}\left(c_{n}\right)\right\|}{\left\|c_{n}\right\|}=0
$$

for $m=1,2, \cdots$.

\section{§2. Quadratic Estimates}

In this section we show that the spectral properties derived above for generators together with a norm estimate on the products of approximate eigenelements can be used to deduce that the only differential operators which are dissipations are quadratic.

Proposition 2.1. Let $\delta$ be a symmetric derivation on a $C^{*}$-algebra $A$ and let $H$ be an operator defined on a *subalgebra $D(H)$ of $D\left(\delta^{p}\right)$, where $p$ is a positive integer, by

$$
H(a)=\sum_{m=0}^{p} \lambda_{m} \delta^{m}(a)
$$

and $\lambda_{m} \in C$. Assume that $H$ is a dissipation, i.e.

$$
H(a * a) \leqq H(a *) a+a * H(a), \quad a \in D(H),
$$

and further assume there exist positive constants $\mu, M$, sequences of real numbers $\left\{k_{n}\right\},\left\{l_{n}\right\}$, and sequences $\left\{a_{n}\right\},\left\{b_{n}\right\}$ of nonzero elements in $D(H)$ such that

$$
\begin{gathered}
\lim _{n \rightarrow \infty}\left|k_{n}\right|=\infty ; \\
\mu \leqq\left|l_{n}\right| \leqq M\left|k_{n}\right|^{1 / 4} ; \\
\left\|\delta^{m}\left(a_{n}\right)-\left(i k_{n}\right)^{m} a_{n}\right\| \leqq \mu\left\|a_{n}\right\|,\left\|\delta^{m}\left(b_{n}\right)-\left(i l_{n}\right)^{m} b_{n}\right\| \leqq \mu\left\|b_{n}\right\|, \\
\left\|\delta^{m}\left(a_{n}^{*} a_{n}\right)\right\| \leqq \mu\left\|a_{n}^{*} a_{n}\right\|,\left\|\delta^{m}\left(b_{n}^{*} b_{n}\right)\right\| \leqq \mu\left\|b_{n}^{*} b_{n}\right\|, \\
\left\|\delta^{m}\left(a_{n}^{*} b_{n}\right)-\left(i\left(l_{n}-k_{n}\right)\right)^{m} a_{n}^{*} b_{n}\right\| \leqq \mu\left\|a_{n}^{*} b_{n}\right\|,
\end{gathered}
$$

for $m=1,2, \cdots, p$ and $n=1,2,3, \cdots$. Moreover assume that

for all $n$.

$$
\left\|a_{n}^{*} b_{n}\right\| \geqq \mu\left\|a_{n}^{*}\right\|\left\|b_{n}\right\|
$$

It follows that $\lambda_{m}=0$ for $m>2$ and $\lambda_{2} \leqq 0$.

If in addition there is a sequence of non-zero elements $c_{n} \in D(H)$ such that

$$
\lim _{n \rightarrow \infty}\left\|\delta\left(c_{n}\right)\right\| /\left\|c_{n}\right\|=0,
$$

it follows that $\lambda_{0} \geqq 0$. define

Proof. Normalize the sequences $a_{n}, b_{n}$, so that $\left\|a_{n}\right\|=1=\left\|b_{n}\right\|$ for all $n$ and 


$$
|\lambda|=\sup _{m}\left|\lambda_{m}\right|
$$

Next if

$$
S(a, b)=H(a *) b+a * H(b)-H_{1} a^{*} b^{\prime}
$$

for $a, b \in D(H)$ then

$$
\omega(S(a, a)) \geqq 0
$$

for all $a \in D(H)$ and all states $\omega$. Hence

$$
|\omega(S(a, b))|^{2} \leqq \omega(S(a, a)) \omega(S(b, b))
$$

by the Cauchy-Schwarz inequality.

Since $\left\|a_{n}^{*} b_{n}\right\| \geqq \mu\left\|a_{n}^{*}\right\|\left\|b_{n}\right\|=\mu$ there exist states $\omega_{n}$ such that

$$
\left|\omega_{n}\left(a_{n}^{*} b_{n}\right)\right| \geqq \mu / 2 .
$$

Now we exploit the Cauchy-Schwarz inequality in the form

$$
\left|\omega_{n}\left(S\left(a_{n}, b_{n}\right)\right)\right|^{2} \leqq \omega_{n}\left(S\left(a_{n}, a_{n}\right)\right) \omega_{n}\left(S\left(b_{n}, b_{n}\right)\right),
$$

for all $n$, to prove $\lambda_{m}=0$ for $m>2$. Define

$$
\begin{aligned}
& T\left(a_{n}, b_{n}\right)=\sum_{m=0}^{p} \lambda_{m}\left\{\left(-i k_{n}\right)^{m}+\left(i l_{n}\right)^{m}-\left(i\left(l_{n}-k_{n}\right)\right)^{m}\right\} a_{n}^{*} b_{n}, \\
& T\left(a_{n}, a_{n}\right)=\sum_{m=0}^{p} \lambda_{m}\left\{\left(-i k_{n}\right)^{m}+\left(i k_{n}\right)^{m}\right\} a_{n}^{*} a_{n}, \\
& T\left(b_{n}, b_{n}\right)=\sum_{m=0}^{p} \lambda_{m}\left\{\left(-i l_{n}\right)^{m}+\left(i l_{n}\right)^{m}\right\} b_{n}^{*} b_{n} .
\end{aligned}
$$

Then the estimates in the hypotheses of the proposition immediately give

$$
\begin{aligned}
& \left\|S\left(a_{n}, b_{n}\right)-T\left(a_{n}, b_{n}\right)\right\| \leqq 3 p|\lambda| \mu, \\
& \left\|S\left(a_{n}, a_{n}\right)-T\left(a_{n}, a_{n}\right)\right\| \leqq 3 p|\lambda| \mu, \\
& \left\|S\left(b_{n}, b_{n}\right)-T\left(b_{n}, b_{n}\right)\right\| \leqq 3 p|\lambda| \mu,
\end{aligned}
$$

and hence

$$
\left|\omega_{n}\left(T\left(a_{n}, b_{n}\right)\right)+O(1)\right|^{2} \leqq\left(\omega_{n}\left(T\left(a_{n}, a_{n}\right)\right)+O(1)\right)\left(\omega_{n}\left(T\left(b_{n}, b_{n}\right)\right)+O(1)\right) .
$$

But

$$
\begin{aligned}
\omega_{n}\left(T\left(a_{n}, b_{n}\right)\right) & =\sum_{m=0}^{p} \lambda_{m}\left\{\left(-i k_{n}\right)^{m}+\left(i l_{n}\right)^{m}-\left(i\left(l_{n}-k_{n}\right)\right)^{m}\right\} \omega_{n}\left(a_{n}^{*} b_{n}\right) \\
& =\lambda_{p} p\left(i l_{n}\right)\left(-i k_{n}\right)^{p-1} \omega_{n}\left(a_{n}^{*} b_{n}\right)+O\left(k_{n}^{p-3 / 2}\right)
\end{aligned}
$$

where $O\left(k_{n}^{p-3 / 2}\right)$ is a polynomial in $l_{n}$ and $k_{n}$ whose coefficients are uniformly bounded in $n$. The estimate follows from $\left|l_{n}\right| \leqq M\left|k_{n}\right|^{1 / 4}$ and the fact that the highest order term of this polynomial is $O\left(k_{n}^{p-2} l_{n}^{2}\right)=O\left(k_{n}^{p-3 / 2}\right)$.

Similarly, 


$$
\omega_{n}\left(T\left(a_{n}, a_{n}\right)\right)=O\left(k_{n}^{p} ;, \omega_{n}\left(T\left(b_{n}, b_{n}\right)\right)=O\left(k_{n}^{p / 4}\right),\right.
$$

and the Cauchy-Schwarz estimate gives

$$
\begin{array}{r}
\left|\lambda_{p}\right|^{2} p^{2}\left|l_{n}\right|^{2}\left|\omega_{n}\left(a_{n}^{*} b_{n}\right)\right|^{2}\left|k_{n}\right|^{2 p-2}+O\left(\left|k_{n}\right|^{2 p-9 / 4}\right) \\
\leqq O\left(\left|k_{n}\right|^{5 p / 4}\right) .
\end{array}
$$

Now since $\left|l_{n}\right|>\mu$ and $\left|\omega_{n}\left(a_{n}^{*} b_{n}\right)\right| \geqq \mu / 2$ for all $n$ it follows in the limit $n \rightarrow \infty$ that one must have $\lambda_{p}=0$ if $2 p-2>(5 / 4) p$, i. e. if $p>8 / 3$. Iterating this argument one deduces that

$$
\lambda_{p}=\lambda_{p-1}=\cdots=\lambda_{3}=0 .
$$

Next we deduce that $\lambda_{2} \geqq 0$ from the estimate $S\left(a_{n}, a_{n}\right) \geqq 0$ which implies $T\left(a_{n}, a_{n}\right) \geqq O(1)$. Thus

$$
\left(2 \lambda_{2}\left(i k_{n}\right)^{2}+\lambda_{0}\right) a_{n}^{*} a_{n} \geqq O(1) .
$$

Evaluating this in a state $\omega_{n}$ with $\omega_{n}\left(a_{n}^{*} a_{n}\right)=1$ one concludes that

and hence $\lambda_{2} \leqq 0$.

$$
-\lambda_{2} k_{n}^{2} \geqq O(1),
$$

Finally if there is a sequence $c_{n} \in D(H)$ of non-zero elements such that

$$
\lim _{n \rightarrow \infty}\left\|\delta\left(c_{n}\right)\right\| /\left\|c_{n}\right\|=0,
$$

then it follows from

that $\lambda_{0} \geqq 0$.

$$
\begin{aligned}
0 & \leqq H\left(c_{n}^{*}\right) c_{n}+c_{n}^{*} H\left(c_{n}\right)-H\left(c_{n}^{*} c_{n}\right) \\
& =\lambda_{0} c_{n}^{*} c_{n}-2 \lambda_{2} \delta\left(c_{n}^{*}\right) \delta\left(c_{n}\right) \\
& =\lambda_{0} c_{n}^{*} c_{n}+O\left(\left\|\delta\left(c_{n}\right)\right\|^{2}\right)
\end{aligned}
$$

The primary conclusion of Proposition 2.1 is that the dissipation property together with spectral conditions forces the differential operator $H$ to be quadratic. The secondary conclusion is that the remaining coefficients satisfy $\lambda_{0} \geqq 0, \lambda_{2} \leqq 0$. But this latter conclusion can be reached for quadratic operators under quite general conditions.

Proposition 2.2. Let $\delta$ be the generator of a non-trivial strongly continuous one-parameter group of *automorphisms of a $C^{*}$-algebra $\mathcal{A}$. Further let $H=\lambda_{0}$ $+\lambda_{1} \delta+\lambda_{2} \delta^{2}$ for some $\lambda_{i} \in C$ and $D(H)=\mathcal{A}_{\infty}$. Then the following conditions are equivalent:

1. $H$ is a dissipation.

2. $\lambda_{0} \geqq 0$ and $\lambda_{2} \leqq 0$.

Proof. If $H$ has the given form, then

$$
H(x *) x+x * H(x)-H(x * x)=\lambda_{0} x * x-2 \lambda_{2} \delta(x *) \delta(x)
$$


and hence $2 \Rightarrow 1$. We shall now prove $1 \Rightarrow 2$.

First since $\delta$ is a generator of a non-trivial group of $*$-automorphisms $\tau$ there exist non-zero elements $c_{n} \in D(\delta)$ such that $\left\|\delta c_{n}\right\| /\left\|c_{n}\right\| \rightarrow 0$ (for example see Remark 1.3). Then it follows from the dissipation inequality, by the calculation above, that

Thus $\lambda_{0} \geqq 0$.

$$
\lambda_{0} c_{n}^{*} c_{n}+O\left(\left\|\delta c_{n}\right\|^{2}\right) \geqq 0
$$

Second noting that the dissipation inequality is equivalent to

$$
\lambda_{0} a * a \geqq 2 \lambda_{2}(\delta a *)(\delta a),
$$

for all $a \in D(H)$, we immediately deduce that if $\lambda_{0}=0$ then $\lambda_{2} \leqq 0$. But if $\lambda_{0}>0$ and $\lambda_{2}>0$ we may assume $\lambda_{0} / 2 \lambda_{2}=1$ by rescaling $\delta$. Then

$$
a * a \geqq(\delta a *)(\delta a)
$$

for all $a \in D(H)$. This immediately implies that $\delta$ is bounded and $\|\delta\| \leqq 1$. Therefore passing to an irreducible representation in which $\delta$ is non-zero we may suppose that $A=B(\mathscr{H})$ and $\delta=a d(i h)$ with $h=h^{*} \in B(\mathscr{H})$. Next choose $2 \times 2$ matrix units in $B(\mathscr{H})$ with respect to which $h$ is not diagonal. This is possible because $\delta$ is non-zero, and so $h$ is not a scalar. Then

$$
h=\left(\begin{array}{ll}
a & b \\
b^{*} & d
\end{array}\right)
$$

with $a=a^{*}, d=d^{*}$, and $b \neq 0$. But the dissipation inequality $e_{12}^{*} e_{12} \geqq\left(\delta e_{12}^{*}\right)\left(\delta e_{12}\right)$ applied with

gives

$$
e_{12}=\left(\begin{array}{ll}
0 & 1 \\
0 & 0
\end{array}\right)
$$

$$
\left(\begin{array}{ll}
0 & 0 \\
0 & 1
\end{array}\right) \geqq\left(\begin{array}{cc}
b b^{*} & * \\
* & *
\end{array}\right),
$$

and this implies $b=0$ which is inconsistent. Therefore $\lambda_{2} \leqq 0$.

\section{§ 3. Applications}

If $\delta$ is unbounded and a generator then it follows from Proposition 1.1 and Remarks 1.2 and 1.3 that the initial hypotheses of Proposition 2.1 are satisfied. It is not, however, evident that the approximate eigenelements $a_{n}, b_{n}$, can always be chosen to satisfy norm estimates

$$
\left\|a_{n}^{*} b_{n}\right\| \geqq \mu\left\|a_{n}^{*}\right\|\left\|b_{n}\right\|,
$$

for some $\mu>0$. In this section we demonstrate that these estimates do indeed follow from a variety of additional hypotheses on $\mathcal{A}$, or on $(\mathcal{A}, \delta$ ). 
First remark that if $A$ is abelian it is relatively easy to use the property $\left\|\left(a^{*}\right)^{m} a^{k}\right\|=\|a\|^{m+k}$ to construct sequences $a_{n}, b_{n}$, satisfying estimates of the above form. This was essentially the method adopted in the proof of Theorem 1.1.B in [2]. We now consider some other cases.

Theorem 3.1. Let $(\mathcal{A}, \boldsymbol{R}, \tau)$ denote a $C^{*}$-dynamical system and let $\delta$ denote the generator of the action $\tau$. Furthermore let $H$ be an operator from $\mathcal{A}_{\infty}$ into $A$ of the form

$$
H=\sum_{m=0}^{p} \lambda_{m} \delta^{m}, \quad \lambda_{m} \in C
$$

and assume $H$ is a dissipation, i.e.

$$
H(a * a) \leqq H(a *) a+a * H(a), \quad a \in \mathcal{A}_{\infty} .
$$

It follows that $\lambda_{n}=0$ for $n>2, \lambda_{2} \leqq 0$, and $\lambda_{0} \geqq 0$, if any of the following four assumptions is fulfilled.

I. There is a factor representation $\pi$ of $\mathcal{A}$ such that the set $\left\{\gamma \in \mathbb{R} ; \pi\left(\mathcal{A}^{\tau}(\gamma)\right) \neq\right.$ $\{0\}\}$ is unbounded.

II. There is a representation $\pi$ of $A$ and a non-zero real number $\gamma$ such that the $\sigma$-weak closure of the linear span of $\pi\left(\mathcal{A}^{\tau}(\gamma) \mathcal{A}^{\tau}(\gamma)^{*}\right)$ contains 1 .

III. There is a factor representation $\pi$ of $\mathcal{A}$, and a strongly continuous one-parameter unitary group $t \in \boldsymbol{R} \mapsto U_{t}=e^{i t K} \in \pi(\mathcal{A})^{\prime \prime}$ such that

a. $\pi\left(\tau_{t}(a)\right)=U_{t} \pi(a) U_{t}^{*}, a \in \mathcal{A}, t \in \boldsymbol{R}$.

b. $K$ is unbounded.

IV. $A$ is simple with identity, and there is an automorphism $\alpha$ of $A$ such that

a. $\alpha \tau_{t}=\tau_{t} \alpha, t \in \boldsymbol{R}$.

b. $\lim _{n \rightarrow \infty}\left\|\left[a, \alpha^{n}(b)\right]\right\|=0, a, b \in \mathcal{A}$.

Proof. By Propositions 1.1 and 2.1 and Remarks 1.2 and 1.3 the proof of the theorem reduces to the construction of approximate eigenelements $a_{n}, b_{n}$ with the property

$$
\left\|a_{n} b_{n}\right\| \geqq \mu\left\|a_{n}\right\|\left\|b_{n}\right\|
$$

for some $\mu>0$. We treat the four cases separately, but first we prove the following lemma which will be used in proving the above estimate in cases I and II. (We will actually only need the lemma in the case that $\pi$ is a faithful representation.)

Lemma 3.2. Let $G$ be a locally compact abelian group, $\alpha$ an action of $G$ on a $C^{*}$-algebra $\mathcal{A}, \pi$ a representation of $\mathcal{A}, \gamma \in \hat{G}$ a character, and $\mathcal{O} \subseteq \hat{G}$ an open 
subset. Assume

$$
\pi\left(A^{\alpha}(0) A^{\alpha}(\gamma)\right) \neq\{0\} .
$$

Then there exist an $x \in \mathcal{A}^{\alpha}(\mathcal{O})$ and $y \in \mathcal{A}^{\alpha}(\gamma)$ such that

$$
\|\pi(x y)\|=\|\pi(x)\|\|\pi(y)\| \neq 0 .
$$

Proof. The only properties of the spaces $\pi\left(A^{\alpha}(\gamma)\right)$ which are used in the proof are

$$
\begin{gathered}
\pi\left(\mathcal{A}^{\alpha}\left(\xi_{1}\right) \mathcal{A}^{\alpha}\left(\xi_{2}\right)\right) \leqq \pi\left(\mathcal{A}^{\alpha}\left(\xi_{1}+\xi_{2}\right)\right) \\
\pi\left(\mathcal{A}^{\alpha}(\xi) *\right)=\pi\left(\mathcal{A}^{\alpha}(-\xi)\right) \\
\pi\left(\mathcal{A}^{\alpha}(0)\right) \text { is a } C^{*} \text {-algebra. }
\end{gathered}
$$

With this in mind we delete the $\pi$ but assume $A$ is represented on a Hilbert space.

Next choose $x^{\prime} \in \mathcal{A}^{\alpha}(\mathcal{O}), y^{\prime} \in \mathcal{A}^{\alpha}(\gamma)$ such that $x^{\prime} y^{\prime} \neq 0$. Then $x^{\prime} y^{\prime} y^{\prime * \neq 0}$. But by spectral theory there then exists a continuous function $f:[0, \infty) \rightarrow[0, \infty)$ such that $f=0$ in a neighbourhood $[0, \varepsilon)$ of the origin and $x^{\prime} f\left(y^{\prime} y^{\prime *}\right) \neq 0$. But

$$
x=x^{\prime} f\left(y^{\prime} y^{\prime *}\right) \in \mathcal{A}^{\tau}(\mathcal{O}) .
$$

Now let $g:[0, \infty) \rightarrow[0, \infty)$ be a continuous function such that $g=0$ in a neighbourhood $\left[0, \varepsilon^{\prime}\right)$ with $\varepsilon^{\prime}<\varepsilon$ but $g(t)=t^{-(1 / 2)}$ for $t \geqq \varepsilon$ and put $y=g\left(y^{\prime} y^{\prime *)} y^{\prime}\right.$. Then $y$ is an approximant to the partial isometry $U$ in the polar decomposition $y=|y| U$ for $y$ and if $P$ is the range projection of $f\left(y^{\prime} y^{\prime *}\right)$ we have $P y=P U$. Hence $x y$ $=x P y=x P U$. But $P$ is contained in the range projection of $U$, which is equal to the range projection of $y^{\prime} y^{\prime *}$. Hence $\|x y\|=\|x\| \cdot\|y\|$.

Remark. Lemma 3.2 can be generalized as follows. If $\mathcal{O}_{1}, \mathcal{O}_{2}$ are open neighbourhoods of $\gamma_{1}, \gamma_{2} \in \hat{G}$, respectively, define

$$
d\left(\mathcal{O}_{1}, \mathcal{O}_{2}\right)=\sup \left\{\frac{\|\pi(x y)\|}{\|\pi(x)\|\|\pi(y)\|} ; x \in \mathcal{A}^{\alpha}\left(\mathcal{O}_{1}\right), y \in \mathcal{A}^{\alpha}\left(\mathcal{O}_{2}\right), \pi(x) \neq 0 \neq \pi(y)\right\} .
$$

Then by a slight variation of the above proof, approximating continuous functions by polynomials, one can show that if

$$
d\left(\gamma_{1}, \gamma_{2}\right) \equiv \inf _{\mathcal{O}_{1}, \mathcal{O}_{2}} d\left(\mathcal{O}_{1}, \mathcal{O}_{2}\right)>0
$$

then

$$
d\left(\gamma_{1}, \gamma_{2}\right)=1
$$

It appears possible that $d\left(\mathcal{O}_{1}, \mathcal{O}_{2}\right)$ can only take the values 0 or 1 and if this were the case Theorem 3.1 would be valid with the spectral condition in I replaced by the assumption that the spectrum of $\tau$ in the representation $\pi$ is unbounded. 
We now return to the proof of Theorem 3.1.

Case I. As $\pi\left(\mathcal{A}^{\tau}(-\gamma)\right)=\pi\left(\mathcal{A}^{\tau}(\gamma)\right) *$, the set $\left\{\gamma \in \mathbb{R}, \pi\left(\mathcal{A}^{\tau}(\gamma)\right) \neq\{0\}\right\}$ is symmetric, and it follows from the hypothesis that there exist sequences $l_{n}^{\prime}, k_{n}^{\prime}$ of positive numbers in this set such that

$$
2 \leqq l_{n}^{\prime} \leqq\left(k_{n}^{\prime}\right)^{1 / 16}-1
$$

and

$$
\lim _{n \rightarrow \infty} k_{n}^{\prime}=+\infty .
$$

Now, as $\pi(\mathcal{A})^{\prime \prime}$ is a factor, the $\sigma$-weak closures of the ideals $\pi\left(\mathcal{A} \mathcal{A}^{\tau}\left(k_{n}^{\prime}\right) \mathcal{A}\right)$ and $\pi\left(\mathcal{A} \mathcal{A}^{\tau}\left(l_{n}^{\prime}\right) \mathcal{A}\right)$ in $\pi(\mathcal{A})$ must be equal to $\pi(\mathcal{A})^{\prime \prime}$. In particular, this implies that

$$
\mathcal{A}^{\tau}\left(k_{n}^{\prime}\right) \mathcal{A} \mathcal{A}^{\tau}\left(l_{n}^{\prime}\right) \neq 0 \text {. }
$$

We will verify the hypotheses of Proposition 2.1 with $\mu=M=1$. First note that, by Proposition 1.1 , for each $k \in \mathbb{R}$ there exists an $\varepsilon=\varepsilon_{k}>0$ such that

$$
\begin{gathered}
\left\|\left.\left(\delta^{m}-\left(i\left(k+k_{n}^{\prime}\right)\right)^{m}\right)\right|_{\mathcal{A}^{\tau}\left(\left[k+k_{n}^{\prime}-\varepsilon, k+k_{n}^{\prime}+\varepsilon\right]\right)}\right\|<1, \\
\left\|\left.\left(\delta^{m}-\left(i\left(k+l_{n}^{\prime}\right)\right)^{m}\right)\right|_{\mathcal{A}^{\tau}\left(\left[k+l_{n}^{\prime}-\varepsilon, k+l_{n}^{\prime}+\varepsilon\right]\right)}\right\|<1, \\
\left\|\left.\left(\delta^{m}-\left(i\left(k+l_{n}^{\prime}+k_{n}^{\prime}\right)\right)^{m}\right)\right|_{\mathcal{A}^{\tau}\left(\left[k+l_{n}^{\prime}+k_{n}^{\prime}-\varepsilon, k+l_{n}^{\prime}+k_{n}^{\prime}+\varepsilon\right]\right)}\right\|<1, \\
\left\|\left.\left(\delta^{m}-(i k)^{m}\right)\right|_{\mathcal{A}^{\tau}([k-\varepsilon, k+\varepsilon])}\right\|<1,
\end{gathered}
$$

and

$$
\left\|\left.\delta^{m}\right|_{\mathcal{A} \tau([-2 s, 2 \varepsilon])}\right\|<1,
$$

for $m=1,2, \cdots, p$. The intervals $\mathcal{O}_{k}=\left(k-\varepsilon_{k}, k+\varepsilon_{k}\right)$ form an open covering of $\boldsymbol{R}$, and so the spectral subspaces $\mathcal{A}^{\tau}\left(\mathcal{O}_{k}\right)$ span all elements in $\mathcal{A}$ with bounded $\tau$-spectrum. It follows that there is a $k_{n}^{\prime \prime} \in \mathbb{R}$ such that

$$
\mathcal{A}^{\tau}\left(k_{n}^{\prime}\right) \mathcal{A}^{\tau}\left(\mathcal{O}_{k_{n}^{\prime \prime}}\right) \mathcal{A}^{\tau}\left(l_{n}^{\prime}\right) \neq 0 \text {. }
$$

We are now going to construct the sequences $a_{n}, b_{n} \in \mathcal{A}_{\infty}, k_{n}, l_{n} \in \mathbb{R}$ satisfying the requirements of Proposition 2.1. There are 5 cases.

Case 1. $\quad k_{n}^{\prime 1 / 4} \leqq k_{n}^{\prime \prime}$. As $\mathcal{A}^{\tau}\left(\mathcal{O}_{k_{n}^{\prime \prime}}\right) \mathcal{A}^{\tau}\left(l_{n}^{\prime}\right) \neq 0$, it follows from Lemma 3.2, applied with $\pi$ equal to any faithful representation of $\mathcal{A}$, that there exist non-zero elements $a_{n} \in \mathcal{A}^{\tau}\left(-\mathcal{O}_{k_{n}^{\prime \prime}}\right), b_{n} \in \mathcal{A}^{\tau}\left(l_{n}^{\prime}\right)$ such that $\left\|a_{n}^{*} b_{n}\right\|=\left\|a_{n}^{*}\right\|\left\|b_{n}\right\|$. If we put $k_{n}=$ $-k_{n}^{\prime \prime}, l_{n}=l_{n}^{\prime}$, we have

$$
2 \leqq l_{n}=l_{n}^{\prime} \leqq k_{n}^{1 / 16} \leqq k_{n}^{\prime 1 / 4}=\left|k_{n}\right|^{1 / 4} .
$$

The spectral-concentration conditions of Proposition 2.1 are satisfied because of the choice of $\varepsilon_{k_{n}^{\prime \prime}}$.

Case 2. $1 \leqq k_{n}^{\prime \prime} \leqq k_{n}^{\prime 1 / 4}$. Then pick non-zero $a_{n} \in \mathcal{A}^{\tau}\left(-k_{n}^{\prime}\right), b_{n} \in \mathcal{A}^{\tau}\left(\mathcal{O}_{k_{n}^{\prime \prime}}\right)$ such that $\left\|a_{n}^{*} b_{n}\right\|=\left\|a_{n}^{*}\right\|\left\|b_{n}\right\|$, and put $k_{n}=-k_{n}^{\prime}, l_{n}=k_{n}^{\prime \prime}$. Then 


$$
1 \leqq l_{n}=k_{n}^{\prime \prime} \leqq k_{n}^{\prime 1 / 4}=\left|k_{n}\right|^{1 / 4}
$$

and the rest of the argument is as in Case 1 .

Case 3. $-1 \leqq k_{n}^{\prime \prime} \leqq 1$. Then, as

$$
\mathcal{A}^{\tau}\left(k_{n}^{\prime}\right) \mathcal{A}^{\tau}\left(\mathcal{O}_{k_{n}^{\prime \prime}}+l_{n}^{\prime}\right) \supseteq \mathcal{A}^{\tau}\left(k_{n}^{\prime}\right) \mathcal{A}^{\tau}\left(\mathcal{O}_{k_{n}^{\prime \prime}}\right) \mathcal{A}^{\tau}\left(l_{n}^{\prime}\right) \neq 0
$$

there exist non-zero $a_{n} \in \mathcal{A}^{\tau}\left(-k_{n}^{\prime}\right), b_{n} \in \mathcal{A}^{\tau}\left(\mathcal{O}_{k_{n}^{\prime \prime}}+l_{n}^{\prime}\right)$ with $\left\|a_{n}^{*} b_{n}\right\|=\left\|a_{n}^{*}\right\|\left\|b_{n}\right\|$. Put $k_{n}=-k_{n}^{\prime}, l_{n}=k_{n}^{\prime \prime}+l_{n}^{\prime}$. Then

$$
1 \leqq l_{n} \leqq 1+l_{n}^{\prime} \leqq 1+k_{n}^{\prime 1 / 16}-1=\left|k_{n}\right|^{1 / 16} \leqq\left|k_{n}\right|^{1 / 4} .
$$

Case 4. $\quad-k_{n}^{\prime 1 / 4} \leqq k_{n}^{\prime \prime} \leqq-1$. Take

$$
a_{n} \in \mathcal{A}^{\mp}\left(-k_{n}^{\prime}\right), b_{n} \in \mathcal{A}^{\tau}\left(\mathcal{O}_{k_{n}^{\prime \prime}}\right), k_{n}=-k_{n}^{\prime}, l_{n}=k_{n}^{\prime \prime} .
$$

Case 5. $k_{n}^{\prime \prime} \leqq-k^{\prime 1 / 4}$. Take

$$
a_{n} \in \mathcal{A}^{*}\left(-\mathcal{O}_{k_{n}^{\prime \prime}}\right), b_{n} \in \mathcal{A}^{\tau}\left(l_{n}^{\prime}\right), k_{n}=-k_{n}^{\prime \prime}, l_{n}=l_{n}^{\prime} .
$$

Note that in all cases, $\left|k_{n}\right| \geqq k_{n}^{\prime}$ and hence $\lim _{n \rightarrow \infty}\left|k_{n}\right|=\infty$. Thus all the hypotheses of Proposition 2.1 are fulfilled, and this ends the proof of Case I.

Case II. It follows from condition II that

$$
1 \in \pi\left(A^{\tau}(\gamma) A^{\tau}(\gamma) A^{\tau}(\gamma) * A^{\tau}(\gamma) *\right)^{\prime \prime}
$$

and by induction

Thus

$$
1 \in \pi\left(\mathcal{A}^{\tau}(\gamma)^{n} \mathcal{A}^{\tau}(\gamma) * n\right)^{\prime \prime}
$$

$$
1 \in \pi\left(\mathcal{A}^{\tau}(n \gamma) \mathcal{A}^{\tau}(n \gamma) *\right)^{\prime \prime}
$$

for $n=1,2, \cdots$. In particular this means that

$$
\mathcal{A}^{\tau}(-n \gamma) y \neq\{0\}
$$

for any $y \in \mathcal{A}$ with $\pi(y) \neq 0$, and $n=1,2, \cdots$. But then it is easy to verify the hypotheses of Proposition 2.1 from Proposition 1.1 and Lemma 3.2, with $k_{n}=$ $-n \gamma$ and $l_{n}$ uniformly bounded. (Again Lemma 3.2 is applied with $\pi$ equal to any faithful representation.)

Case III. Note that the hypothesis III implies that $\tau_{t}$ passes to a strongly continuous one-parameter group $\tau^{\pi}$ of *automorphisms of $\pi(\mathcal{A})$, and hence $\delta$ passes to a derivation $\delta_{\pi}$ of $\pi(\mathcal{A})$ such that

$$
\delta_{\pi}(\pi(a))=\pi(\delta(a)), \quad a \in D(\delta) .
$$

Then $\delta_{\pi}$ is the generator of $\tau^{\pi}$. Now, by [7], Théorème 3.3 any element of $A_{\infty}$ is a linear combination of elements $\int_{\boldsymbol{R}} d t f(t) \tau_{t}(a)$ where $f \in C_{00}^{\infty}(\boldsymbol{R})$ and $a \in \mathcal{A}$. It 
follows immediately that

$$
\pi(\mathcal{A})_{\infty}=\pi\left(\mathcal{A}_{\infty}\right)
$$

where $\pi(A)_{\infty}$ denotes the $C_{\infty}$-elements for the group $\tau^{\pi}$. Thus, if we define $H_{\pi}$ on $\pi(\mathcal{A})_{\infty}$ by

$$
H_{\pi}=\sum_{m=0}^{p} \lambda_{m} \delta_{\pi}^{m}
$$

then

$$
H_{\pi} \circ \pi=\pi \circ H
$$

and thus $H_{\pi}$ is a dissipation.

The conclusion of these remarks is that we may assume that $\pi$ is faithful in case III, and we will assume this from now on. We also suppress the notation $\pi$, and assume that $\mathcal{A}$ is faithfully represented on a Hilbert space $\mathscr{H}$ in a representation with the relevant properties.

As $\tau$ is unitarily implemented, $\tau$ extends to a $\sigma$-weakly continuous oneparameter group of inner *-automorphisms of $\mathscr{M}=\mathcal{A}^{\prime \prime}$. Let $\mathscr{M}_{\infty}$ be the $C^{\infty}$-elements of this extended action. Then $\mathscr{M}_{\infty}$ is a Fréchet space in the topology defined by the seminorms $x \mapsto\left\|\delta^{n} x\right\|, n=0,1,2, \cdots$.

Observation 1. If $x \in \mathscr{M}_{\infty}$, then there exists a net $x_{m} \in \mathcal{A}_{\infty}$ such that

$$
\delta^{n}(x)=\lim _{m \rightarrow \infty} \delta^{n}\left(x_{m}\right)
$$

for $n=0,1,2, \cdots$, where the limit is in the $\sigma$-strong* operator topology on $\mathscr{M}$, and the net $m \mapsto\left\|\delta^{n}\left(x_{m}\right)\right\|$ is uniformly bounded for each $n$.

Proof. The group $\tau$ restricts to a one-parameter group of *-automorphisms of $\mathscr{M}_{\infty}$, and as

$$
\left\|\delta^{n}\left(\tau_{t}(x)-x\right)\right\|=\left\|\int_{0}^{t} d s \delta^{n+1} \tau_{s}(x)\right\| \leqq|t|\left\|\delta^{n+1}(x)\right\|
$$

this restricted group is continuous in the Fréchet topology on $\mathscr{N}_{\infty}$. It follow's from [7], Théorème 3.3 that any $x \in \mathscr{M}_{\infty}$ has the form

$$
x=\sum_{k} \int_{-\infty}^{\infty} d t f_{k}(t) \tau_{t}\left(y_{k}\right)=\sum_{k} \tau_{f_{k}}\left(y_{k}\right)
$$

where $y_{k} \in \mathscr{M}_{\infty}$ and $f_{k} \in C_{00}^{\infty}(\boldsymbol{R})$ and the sum is finite. By Kaplansky's density theorem, [5], Theorem 2.4.16, there are uniformly bounded nets $m \mapsto y_{k, m} \in \mathcal{A}_{\infty}$ converging $\sigma$-strongly to $y_{k}$, and we may use the same index set $\{m\}$ for all the nets. Put

$$
x_{m}=\sum_{k} \tau_{f_{k}}\left(y_{k, m}\right) .
$$

Then $x_{m} \in \mathcal{A}_{\infty}$, and

$$
\delta^{n}\left(x_{m}\right)=(-1)^{n} \sum_{k} \tau_{f_{k}^{(n)}}\left(y_{k, m}\right)
$$


is uniformly bounded in $m$ for each $n$. Furthermore

$$
\begin{aligned}
\lim _{m \rightarrow \infty} \delta^{n}\left(x_{m}\right) & =(-1)^{n} \sum_{k} \lim _{m} \tau_{f_{k}^{(n)}}\left(y_{k, m}\right) \\
& =(-1)^{n} \sum_{k} \tau_{f_{k}^{(n)}}\left(y_{k}\right) \\
& =\delta^{n}(x),
\end{aligned}
$$

where the limits are in the $\sigma$-strong* topology, and we have used the following result.

Observation 2. If $\tau$ is a $\sigma$-weakly continuous one-parameter group of *automorphisms of a von Neumann algebra $\mathscr{M}$, the maps $\tau_{f}$, where $f \in C_{00}^{\infty}(\boldsymbol{R})$, are $\sigma$-strong*- $\sigma$-strong* continuous on the unit sphere of $\mathscr{M}$.

Proof. As $\left(\tau_{f}(x)\right) *=\tau_{\bar{f}}\left(x^{*}\right)$ it is enough to check that $\tau_{f}$ is $\sigma$-strong- $\sigma$-strong continuous on the unit sphere, and realizing $\mathscr{M}$ in a standard representation on a Hilbert space $\mathscr{H}$ we may assume that $\tau$ is implemented by a strongly continuous unitary group $U$ and that all the seminorms defining the $\sigma$-strong topology have the form $x \mapsto\|x \xi\|$, where $\xi \in \mathcal{H}$. Now, if $x_{m}$ is a norm-bounded net converging to $x \sigma$-strongly we have to show

$$
\int_{-\infty}^{\infty} d t f(t) U_{t} x_{m} U_{t}^{*} \xi \underset{m \rightarrow \infty}{\longrightarrow} \int_{-\infty}^{\infty} d t f(t) U_{t} x U_{t}^{* \xi} .
$$

But since $\left\|f(t) U_{t} x_{m} U_{t}^{*} \xi-f(t) U_{t} x U_{t}^{*} \xi\right\| \leqq ! f(t) \mid\left\|\left(x_{m}-x\right) U_{t}^{*} \xi\right\|$, and $\lim _{m \rightarrow \infty}\left\|\left(x_{m}-x\right) U_{t}^{*} \xi\right\|$ $=0$ for each $t$, and the family of functions $t \rightarrow\left\|\left(x_{m}-x\right) U_{t}^{*} \xi\right\|$ is equicontinuous in $m$, it follows that $\left\|\left(x_{m}-x\right) U_{t}^{*} \xi\right\| \rightarrow 0$ uniformly on the compact support of $f$, and hence

$$
\lim _{m \rightarrow \infty} \tau_{f}\left(x_{m}\right) \xi=\tau_{f}(x) \xi .
$$

Turning back to the proof of Case III of Theorem 3.1, we extend $H$ to $\mathscr{M}_{\infty}$ by setting

$$
H(x)=\sum_{m=0}^{p} \lambda_{m} \delta^{m}(x)
$$

for $x \in \mathscr{M}_{\infty}$, where $\delta$ now denotes the generator of the extension of $\tau$ to $\mathscr{M}$. If $x \in \mathscr{M}_{\infty}$ it follows from Observation 1 that there is a bounded net $x_{m} \in \mathcal{A}_{\infty}$ such that $\delta^{n}\left(x_{m}\right) \rightarrow \delta^{n}(x) \quad \sigma$-strongly* for $n=0,1, \cdots$. But then it follows from the derivation property of $\delta$ and the explicit form of $H$ given above that

$$
\begin{aligned}
& H\left(x_{m}^{*} x_{m}\right) \underset{m \rightarrow \infty}{\longrightarrow} H\left(x^{*} x\right) \\
& H\left(x_{m}^{*}\right) x_{m} \underset{m \rightarrow \infty}{\longrightarrow} H\left(x^{*}\right) x \\
& x_{m}^{*} H\left(x_{m}\right) \underset{m \rightarrow \infty}{\longrightarrow} x * H(x
\end{aligned}
$$


where the limits are now in the $\sigma$-weak topology. Since $\left.H\right|_{A_{\infty}}$ is a dissipation it follows by limiting that $H$ is a dissipation on $\mathscr{M}_{\infty}$.

Now, by adding a multiple of the identity to the self-adjoint operator $K$ if necessary, we may assume $0 \in \operatorname{Spectrum}(K)$. Let $k_{n}$ be a sequence in $\operatorname{Spectrum}(K)$ such that $\left|k_{n}\right| \geqq\left|k_{1}\right| \geqq 1$ for all $n=1,2, \cdots$, and $\lim _{n \rightarrow \infty}\left|k_{n}\right|=\infty$. Let $\varepsilon_{n}$ be a sequence of positive reals such that

and

$$
\begin{aligned}
& \left\|\left(\delta^{m}-\left(i k_{n}\right)^{m}\right) \mid \operatorname{Ii\tau }_{\tau}\left(\left[k_{n}-2 i_{n} \cdot k_{n}+2 \varepsilon_{n}\right]\right)\right\|<1, \\
& \left\|\left(\delta^{m}-\left(i\left(k_{1}-k_{n}\right)\right)^{m}\right) \mid \operatorname{Sit}_{i}\left(\left[k_{1}-k_{n}-2 \varepsilon_{n} k_{1}-k_{n}+9 \varepsilon_{n}\right]\right)\right\|<1, \\
& \left\|\delta^{m} \mid \Omega^{\prime}=\left(\left[-2 \varepsilon_{n} s \varepsilon_{n}\right]\right)\right\|<1 \text {, }
\end{aligned}
$$

$$
\varepsilon_{n} \leqq \varepsilon_{1}
$$

for $m=1,2, \cdots, p$. (These $\varepsilon_{n}$ 's exist by Proposition 1.1 ; the problem that $\tau$ is a weakly continuous group on a von Neumann algebra rather than a strongly continuous group on a $C^{*}$-algebra can for example be avoided by restricting to the strong continuity subspace $\mathscr{M}_{0}$ for $\tau$,

$$
\begin{aligned}
\mathscr{N}_{0} & =\left\{x \subset \mathscr{M}_{i} ; t \longmapsto \tau_{t}(x) \text { is norm continuous }\right\} \\
& =\text { Norm closure of } D(\delta) \\
& \left.=\text { Norm closure of } \bigcup_{n} \mathscr{M}^{\tau}([-n, n]) .\right)
\end{aligned}
$$

Let $E$ denote the projection valued spectral measure of $K$. Then

$$
E\left(\left[-\varepsilon_{n}, \varepsilon_{n}\right]\right) \mathscr{M} E\left(\left[-k_{n}-\varepsilon_{n},-k_{n}+\varepsilon_{n}\right]\right) \subseteq \mathscr{M}^{\tau}\left(\left[k_{n}-2 \varepsilon_{n}, k_{n}+2 \varepsilon_{n}\right]\right)
$$

by [5], Proposition 3.2.43. Since $\mathscr{M}$ is a factor, the left side of this relation is non-zero. Also, if

$$
x \in E\left(\left[-\varepsilon_{n}, \varepsilon_{n}\right]\right) \mathcal{M E}\left(\left[-k_{n}-\varepsilon_{n},-k_{n}+\varepsilon_{n}\right]\right)
$$

and $x=U|x|$ is the polar decomposition of $x$, then $E\left(\left[-\varepsilon_{n}, \varepsilon_{n}\right]\right) U=U=$ $U E\left(\left[-k_{n}-\varepsilon_{n},-k_{n}+\varepsilon_{n}\right]\right)$ and thus

$$
U \in E\left(\left[-\varepsilon_{n}, \varepsilon_{n}\right]\right) \operatorname{ML} E\left(\left[-k_{n}-\varepsilon_{n},-k_{n}+\varepsilon_{n}\right]\right) .
$$

In particular there exist non-zero partial isometries

$$
\begin{aligned}
b_{n} & \in E\left(\left[-\varepsilon_{n}, \varepsilon_{n}\right]\right) \mathscr{M} E\left(\left[-k_{1}-\varepsilon_{n},-k_{1}+\varepsilon_{n}\right]\right) \\
& \cong \mathscr{M}^{\tau}\left(\left[k_{1}-2 \varepsilon_{n}, k_{1} \div 2 \varepsilon_{n}\right]\right)
\end{aligned}
$$

and for the same reason there exist non-zero partial isometries

$$
\begin{aligned}
a_{n} & \in b_{n} b_{n}^{*} \mathscr{M} E\left(\left[-k_{n}-\varepsilon_{n},-k_{n}+\varepsilon_{n}\right]\right) \\
& \cong E\left(\left[-\varepsilon_{n}, \varepsilon_{n}\right]\right) \mathscr{M} E\left(\left[-k_{n}-\varepsilon_{n},-k_{n}+\varepsilon_{n}\right]\right) \\
& \cong M^{=}\left(\left[k_{n}-2 \varepsilon_{n}, k_{n}+2 \varepsilon_{n}\right]\right) .
\end{aligned}
$$


But then $a_{n} a_{n}^{*} \leqq b_{n} b_{n}^{*}$ and hence

$$
\left\|a_{n}^{*} b_{n}\right\|=1=\left\|a_{n}^{*}\right\|\left\|b_{n}\right\| \text {. }
$$

Now, putting $l_{n}=k_{1}$ for $n=1,2, \cdots$ it follows from

$$
\begin{aligned}
& b_{n} \in \mathscr{M}^{\tau}\left(\left[k_{1}-2 \varepsilon_{1}, k_{1}+2 \varepsilon_{1}\right]\right), \\
& a_{n}^{*} \in \mathscr{M}^{\tau}\left(\left[-k_{n}-2 \varepsilon_{n},-k_{n}+2 \varepsilon_{n}\right]\right), \\
& a_{n}^{*} b_{n} \subseteq \mathscr{M} E\left(\left[-k_{n}-\varepsilon_{n},-k_{n}+\varepsilon_{n}\right]\right) \mathscr{M} E\left(\left[-k_{1}-\varepsilon_{n},-k_{1}+\varepsilon_{n}\right]\right) \\
& \cong \mathscr{M}^{\tau}\left(\left[k_{1}-k_{n}-2 \varepsilon_{n}, k_{1}-k_{n}+2 \varepsilon_{n}\right]\right), \\
& a_{n}^{*} a_{n} \in E\left(\left[-k_{n}-\varepsilon_{n},-k_{n}+\varepsilon_{n}\right]\right) \mathscr{M} E\left(\left[-k_{n}-\varepsilon_{n},-k_{n}+\varepsilon_{n}\right]\right) \\
& \cong \mathscr{M}^{\tau}\left(\left[-2 \varepsilon_{n}, 2 \varepsilon_{n}\right]\right)
\end{aligned}
$$

and

$$
b_{n}^{*} b_{n} \in \mathscr{M}^{\tau}\left(\left[-2 \varepsilon_{n}, 2 \varepsilon_{n}\right]\right)
$$

that all the estimates of Proposition 2.1 are fulfilled, with $\mu=1, M=\left|k_{1}\right|$. This ends the proof of Case III.

Case IV. It follows from [8] Lemma 2.2 that

$$
\lim _{n \rightarrow \infty}\left\|a \alpha^{n}(b)\right\|=\|a\|\|b\|, \quad a, b \in \mathcal{A} .
$$

But since $\alpha$ commutes with $\tau$ one has $\alpha\left(\mathcal{A}^{\tau}(K)\right)=\mathcal{A}^{\tau}(K)$ for any subset $K \leqq \boldsymbol{R}$. Therefore if $K_{1}, K_{2}$ are closed subsets of $\boldsymbol{R}$ with $\mathcal{A}^{\tau}\left(K_{i}\right) \neq\{0\}$ for $i=1,2$, then $\mathcal{A}^{\tau}\left(K_{1}+K_{2}\right) \neq\{0\}$. Moreover

$$
\sup \left\{\frac{\|a b\|}{\|a\|\|b\|} ; a \in \mathcal{A}^{\tau}\left(K_{1}\right), b \in \mathcal{A}^{\tau}\left(K_{2}\right), a \neq 0 \neq b\right\}=1 .
$$

Therefore, if $\delta$ is non-zero, the spectrum of $\tau$, and of $\delta$, is a group and hence is unbounded. Furthermore one can construct sequences $a_{n}, b_{n}$, with the properties of Proposition 2.1 for any $0<\mu<1$. On the other hand the case $\delta=0$ is trivial.

\section{§4. Multiparameter Actions}

Let $\tau$ be an action of $R^{\nu}$ on a $C^{*}$-algebra $\mathcal{A}$, where $\nu=2,3, \cdots$, and let $\delta_{1}, \cdots, \delta_{\nu}$ be the generators of this action in the $\nu$ coordinate directions. If $\alpha=$ $\left(\alpha_{1}, \alpha_{2}, \cdots, \alpha_{\nu}\right)$ is a multi-index consisting of non-negative integers, define $|\alpha|=$ $\alpha_{1}+\alpha_{2}+\cdots+\alpha_{\nu}$ and

$$
\delta^{\alpha}=\delta_{1}^{\alpha_{1}} \cdots \delta_{\nu}^{\alpha_{\nu}} .
$$

In [2] it was proved that if $\mathcal{A}$ is abelian and the action $\tau$ is free, then a polynomial operator $H$ of the form 


$$
H=\sum_{\alpha} l_{\alpha} \delta^{\alpha}
$$

where $l_{\alpha}$ are continuous functions on the spectrum $X$ of $A$ is a dissipation if, and only if, $l_{\alpha}=0$ for $|\alpha|>2$, the $\nu \times \nu$ matrix $\left[l_{i j}(\omega)\right]$ is negative definite for all $\omega \in X$ where

and

$$
l_{i j}= \begin{cases}(1 / 2) l_{\alpha}, \text { where } \alpha_{i}=\alpha_{j}=1, \alpha_{k}=0 & \text { for } k \neq i, j, \text { if } i \neq j \\ l_{\alpha}, \text { where } \alpha_{i}=2, \alpha_{k}=0 & \text { for } k \neq i, \text { if } i=j\end{cases}
$$

$$
l_{(0,0, \cdots, 0)}(\omega) \geqq 0
$$

for all $\omega$. This result has some extensions to the case of non-abelian $\mathcal{A}$, but the analogue of Proposition 2.1 becomes much more complicated in the general setting. One has to assume that $\tau$ has unbounded spectrum in sufficiently many directions. However, an analogue of Theorem 3.1 in the cases II and IV is easier to formulate and prove, since either of these conditions implies that the spectrum, or part of the pointspectrum, of $\tau$ is a subgroup of $R^{\nu}$, and in case IV one has

$$
\sup \left\{\frac{\|a b\|}{\|a\|\|b\|} ; a \in \mathcal{A}^{\tau}\left(\mathcal{O}_{1}\right), b \in \mathcal{A}^{\tau}\left(\mathcal{O}_{2}\right), a \neq 0 \neq b\right\}=1
$$

whenever $\mathcal{O}_{1}, \mathcal{O}_{2}$ are subsets of $\boldsymbol{R}^{\nu}$ such that $\mathcal{A}^{\tau}\left(\mathcal{O}_{i}\right) \neq 0$ for $i=1,2$. Using this, and techniques from the proof of Proposition 2.1 and from [2], it is not hard to establish the following result.

Theorem 4.1. Let $\left(\mathcal{A}, \boldsymbol{R}^{\nu}, \tau\right)$ denote a $C^{*}$-dynamical system and let $H$ be an operator from $\mathcal{A}_{\infty}$ into $\mathcal{A}$ which is a polynomial in the products $\delta^{\alpha}$ of $\delta_{1}, \cdots, \delta_{\nu}$ with constant coefficients. Assume that $H$ is a dissipation, i.e.

$$
H(a * a) \leqq H(a *) a+a * H(a), \quad a \in \mathcal{A}_{\infty} .
$$

It follows that $H$ has the form

$$
H(a)=\sum_{i, j=1}^{\nu} \lambda_{i j} \delta_{\imath} \delta_{\jmath}(a)+\sum_{i=1}^{\nu} \lambda_{\imath} \delta_{\imath}(a)+\lambda_{0} a,
$$

where $\left[\lambda_{i j}\right]$ is a real, negative-definite matrix and $\lambda_{0} \geqq 0$, if either of the following two assumptions is fulfilled.

I. There are a representation $\pi$ of $A$ and non-zero elements $\gamma_{1}, \cdots, \gamma_{\nu}$ in $\mathbb{R}^{\nu}$ such that

a. The $\sigma$-weak closure of the linear span of $\pi\left(\mathcal{A}^{\tau}\left(\gamma_{i}\right) \mathcal{A}^{\tau}\left(\gamma_{i}\right)^{*}\right)$ contains I for $i=1, \cdots, \nu$.

b. The linear span of $\left\{\gamma_{1}, \cdots, \gamma_{\nu}\right\}$ is $\mathbb{R}^{\nu}$.

II. $A$ is simple, with identity, and there is an automorphism $\alpha$ of $A$ such that a. $\alpha \circ \tau_{\tau}=\tau_{t} \circ \alpha$, for all $t \in \mathbb{R}^{\nu}$. 
b. $\lim _{n \rightarrow \infty}\left\|\left[a, \alpha^{n}(b)\right]\right\|=0$, for all $a, b \in \mathcal{A}$.

The details of the proof are left to the reader. Note that in Case I the representation

$$
H=\sum_{i, \jmath=1}^{\nu} \lambda_{\imath \jmath} \delta_{\imath} \delta_{\jmath}+\sum_{i=1}^{\nu} \lambda_{\imath} \delta_{\imath}+\lambda_{0}
$$

is unique, and in Case II the representation is unique if, and only if, the linear span of the spectrum of $\tau$ is all of $\mathbb{R}^{\nu}$.

\section{$\S 5$. Derivations}

It is a simple consequence of Proposition 2.1 and Theorem 3.1 that if in addition to the other assumptions $H$ is a derivation, then it has the form $H=\lambda \delta$ (in Theorem 4.1, $H=\sum_{j=1}^{\nu} \lambda_{j} \delta_{\jmath}$ ). It is, however, not true in general that a polynomial in a derivation $\delta$ is a derivation only if it is linear in $\delta$, in the same way that a dissipation which is a polynomial in $\delta$ is not necessarily of second order (see Example 0.1). As an example let $\mathcal{A}=M_{n}$, the algebra of all $n \times n$ matrices, and let $\delta=\operatorname{ad}(i d), H=\operatorname{ad}(i h)$, where $d, h$ are diagonal self-adjoint matrices with eigenvalues $d_{1}, \cdots, d_{n}$ and $h_{1}, \cdots, h_{n}$, respectively. Then, as the spectrum of $\delta$ and $H$ are finite, $H$ is a polynomial in $\delta$ if, and only if, $H$ is a function of $\delta$, and it is easy to check that this is the case if, and only if,

$$
h_{\imath}-h_{\jmath}=f\left(d_{i}-d_{\jmath}\right)
$$

for all pairs $i, j$. But this is true if, and only if, $d_{i}-d_{\jmath}=d_{\imath^{\prime}}-d_{\jmath^{\prime}}$ implies $h_{\imath}-h_{\jmath}$ $=h_{i^{\prime}}-h_{j^{\prime}}$ for all $i, j, i^{\prime}, j^{\prime}$. Thus if all the differences $d_{\imath}-d_{j}$ are distinct when $i \neq j$, then $H$ is automatically a polynomial in $\delta$ whatever the values of $h_{1}, \cdots, h_{n}$, so if $n \geqq 3$ there are examples of derivations $H$ which are polynomials in $\delta$ without being linear functions.

\section{Acknowledgements}

This work was begun whilst two of the authors (O. B. and G. E.) were visiting the Australian National University in Canberra with financial support from the Mathematical Sciences Research Centre. The main part of the work was done whilst O. B. and D. W. R. were guests of the Mathematical Sciences Research Institute, Berkeley, and it was completed whilst O. B. was again visiting ANU with support from the Mathematical Sciences Research Centre. We are indebted to A. Kishimoto for pointing out several mistakes in the preprint version of this paper. 


\section{References}

[1] Bratteli, O. and Evans, D.E., Derivations Tangential to Compact Groups; The non-Abelian Case. Proc. Lon. Math. Soc., 52 (1986), in press.

[2] Bratteli, O., Elliott, G. A. and Robinson D.W., The Characterization of Differential Operators by Locality: Classical Flows. Compositio Math., to appear.

[3] Bratteli, O. and Goodman, F., Derivations Tangential to Compact Group Actions: Spectral Conditions in the weak closure. Canad. J. Math., 37 (1985), 160-192.

[4] Bratteli, O. and Jørgensen, P.E.T., Derivations Commuting with Abelian Gauge Actions on Lattice Systems. Commun. Math. Phys. 87 (1982), 353-364.

[5] Bratteli, O. and Robinson, D.W., Operator Algebras and Quantum Statistical Mechanics Vol. I. Springer-Verlag (1979).

[6] Connes, A., Une Classification des Facteurs de Type III, Ann. Sci. Ecole Norm. Sup. 6 (1973), 133-252.

[7] Dixmier, J. and Malliavin, P., Factorisations de functions et de vecteurs indéfiniment différentiables, Bull. Sci. Math., $2^{\mathrm{e}}$ séries 102 (1978), 305-330.

[8] Kishimoto, A. and Robinson, D.W., Dissipations, Derivations, and Asymptotic Abelianness. J. Operator Theory 13 (1985), 237-253.

[9] Rudin, W., Fourier Analysis on Groups. Interscience Publishers (1967). 
